\title{
Non-Markovian spontaneous emission from a single quantum dot
}

Madsen, Kristian Høeg; Ates, Serkan; Lund-Hansen, Toke; Löffler, Andreas; Reitzenstein, Stephan; Forchel, Alfred; Lodahl, Peter

Published in:

Proceedings of Frontiers in Optics (FiO) 2011/Laser Science (LS) XXVII conference

Publication date:

2011

Link back to DTU Orbit

Citation (APA):

Madsen, K. H., Ates, S., Lund-Hansen, T., Löffler, A., Reitzenstein, S., Forchel, A., \& Lodahl, P. (2011). NonMarkovian spontaneous emission from a single quantum dot. In Proceedings of Frontiers in Optics (FiO) 2011/Laser Science (LS) XXVII conference (pp. FThE2). Optical Society of America. http://www.frontiersinoptics.com/home.aspx

\section{General rights}

Copyright and moral rights for the publications made accessible in the public portal are retained by the authors and/or other copyright owners and it is a condition of accessing publications that users recognise and abide by the legal requirements associated with these rights.

- Users may download and print one copy of any publication from the public portal for the purpose of private study or research.

- You may not further distribute the material or use it for any profit-making activity or commercial gain

- You may freely distribute the URL identifying the publication in the public portal

If you believe that this document breaches copyright please contact us providing details, and we will remove access to the work immediately and investigate your claim. 


\title{
Non-Markovian spontaneous emission from a single quantum dot
}

\author{
Kristian H. Madsen, Serkan Ates, and Toke Lund-Hansen \\ DTU Fotonik, Department of Photonics Engineering, Technical University of Denmark, Ørsteds Plads 343, DK-2800 \\ Kgs. Lyngby, Denmark \\ khoe@fotonik.dtu.dk \\ Andreas Löffler, Stephan Reitzenstein, and Alfred Forchel \\ Technische Physik, Universität Würzburg, Am Hubland, D-97074 Würzburg, Germany \\ Peter Lodahl \\ DTU Fotonik, Department of Photonics Engineering, Technical University of Denmark, Ørsteds Plads 343, DK-2800 \\ Kgs. Lyngby, Denmark
}

\begin{abstract}
We observe non-Markovian dynamics of a single quantum dot when tuned into resonance with a cavity mode. Excellent agreement between experiment and theory is observed providing the first quantitative description of such a system.

(C) 2011 Optical Society of America

OCIS codes: $270.5580,350.4238$
\end{abstract}

\section{Introduction}

All-solid-state cavity quantum electrodynamics (CQED) systems based on quantum dots (QDs) in nanophotonic cavities provide a promising platform for practical implementations of quantum information protocols [1]. Solid-state systems are of inherently manybody nature, and a number of unique properties distinguish QD-based QED systems from their atomic counterparts including the presence of multiexciton states and deviations from the point-dipole emitter description. The ability to enter the coherent-coupling regime was demonstrated by recording detuning-dependent emission spectra [2]. This proves that the QD-cavity coupling is so strong that there is 'memory' in the system, i.e. the population of the QD at one instant of time depends on its value at previous times, which leads to creation of light-matter entanglement. These so-called non-Markovian effects of the photon-matter coupling appear in general when the local density of optical states (LDOS) is varying rapidly in frequency relative to the QD linewidth.

\section{Detuning Dependent Time-Resolved Measurements}

The coupled QD-cavity system is described by the widely used dissipative Jaynes-Cummings (J-C) model, which is characterized by the following parameters: the cavity decay rate $\kappa=\omega_{c a} / Q$, the QD decay rate $\gamma$ due to the spontaneous emission out of the cavity, the coupling strength $g$, the pure dephasing rate $\gamma_{d p}$, and the cavity-QD frequency detuning $\Delta=\omega_{c a}-\omega_{q d}$. Our system under study consists of a single QD coupled to the fundamental mode of a micropillar cavity. Figure 1(a) shows a typical low temperature emission spectrum of the system under quasi-resonant excitation of the QD. The single emitter nature of the excited QD has been verified by pulsed autocorrelation measurements using a Hanbury Brown-Twiss type interferometer, and for a large detuning we obtain $g^{(2)}(0)=13.2 \%$. In order to have a quantitative comparison to the model, all parameters describe the system were independently measured. First, the quality factor of the cavity is obtained by performing above band pumping under a strong excitation power to ensure all QDs are saturated. By deconvoluting the spectrum with the spectrometer resolution, we find $Q=12200 \pm 410$ corresponding to $\kappa=166.7 \mathrm{~ns}^{-1}$. Second, the dynamics of the QD emission was investigated by time-resolved photoluminescence measurements under systematic variation of the detuning $\Delta$ via temperature tuning. The decay rate of the QD has been extracted as $\gamma=1.94 \mathrm{~ns}^{-1}$ under large detuning conditions. Third, the coherence of the emitted single photons has been measured with a Hong-Ou-Mandel interferometer [3]. The photon wavepacket overlap $V$ determines the degree of indistinguishability of the emitted single photons, and we measure $V=48 \%$. The reduction in $V$ is due to dephasing of the photon wavepacket primarily by phonons. From the measured overlap we extract the pure dephasing rate of the QD and obtain $\gamma_{d p}=(9.6 \pm 3.3) \mathrm{ns}^{-1}$ at $T=16.3 \mathrm{~K}$. Finally, the coupling strength $\mathrm{g}$ can be determined 

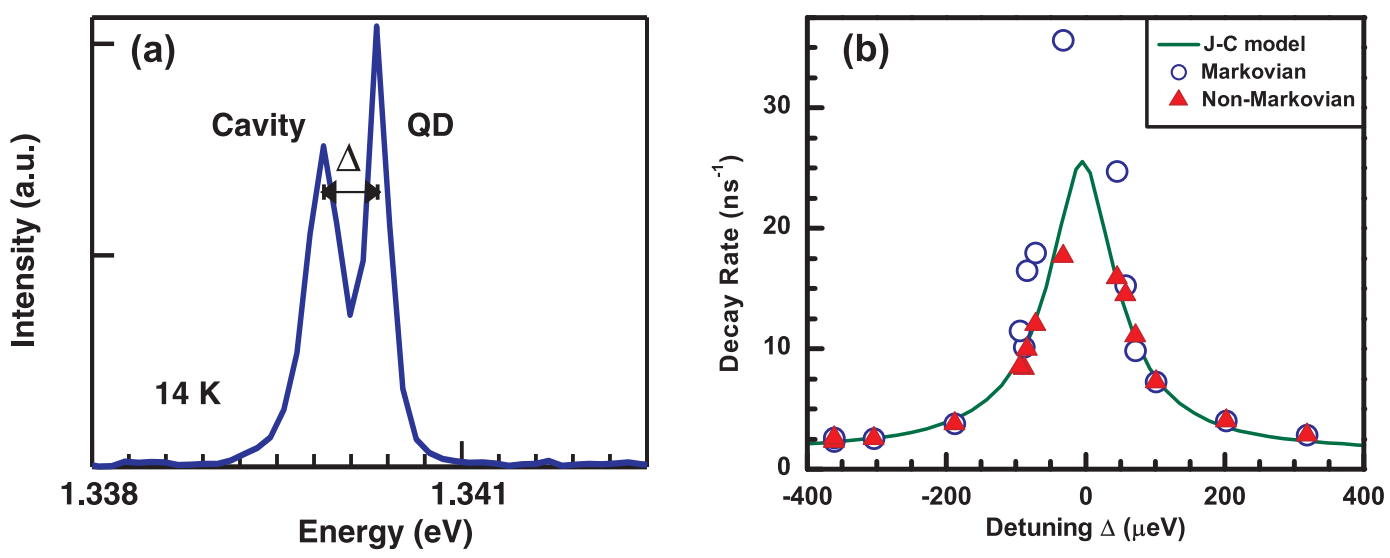

Fig. 1. a) Example of an emission spectrum obtained with p-shell excitation at $T=14 \mathrm{~K}$. b) The detuning-dependent decay rate of the QD extracted by assuming the Markov approximation and with no approximations (non-Markovian). The large deviation between the two approaches clearly illustrates the importance of non-Markovian effects. The theory curve is calculated using only the experimentally measured parameters and no free fitting parameters [4].

unambiguously from detuning dependent time-resolved measurements. Close to resonance, the decay curves are fitted with the solution obtained from the J-C model, where the experimentally measured values of $\gamma, \kappa$, and $\gamma_{d p}$ are used. Additionally, a slow component with decay rate $\gamma_{s}$ accounts for dark exciton recombination and the theory is convoluted with the measured instrument response function. We extract the coupling strength $g=34.3 \pm 1.4 \mathrm{~ns}^{-1}$, which shows that the system is close to the coherent coupling regime, where non-Markovian effects can play a role.

In order to quantify the deviation from Markovian dynamics, we extract the mean decay rate $1 /\langle t\rangle=\int_{0}^{\infty} \rho_{q d} d t / \int_{0}^{\infty} t$. $\rho_{q d} d t$ from the multi-exponential decay curves observed close to resonance. For comparison, we also extract the exponential rate within the Markov approximation, where the fast components of the decay curves are modeled by a single-exponential function. The comparison of the data with and without the Markov approximation is shown in Figure 1(b) for all values of the QD-cavity detuning. As it is seen, a pronounced deviation close to resonance is observed, which indicates the breakdown of the Markov approximation. An important consequence of this deviation is unambiguously reflected in the value of the decay rate at resonance, which is over estimated as $35.6 \mathrm{~ns}^{-1}$ within the Markov approximation while the correct value of the mean decay rate in the full non-Markovian model is $17.7 \mathrm{~ns}^{-1}$. This large discrepancy clearly demonstrates the importance of including non-Markovian effect in the analysis [4].

\section{Conclusion}

We have observed non-Markovian dynamics of a QD coupled to a micropillar cavity. The non-Markovian dynamics is observed close to cavity resonance, and the experimental signature is multi-exponential decay dynamics. Failing to account for non-Markovian effects were found to lead to a large overestimate of the coupling strength. Finally, we observed excellent agreement between theory and experiment for the detuning-dependent decay rates without assuming any free parameters, thus providing the first quantitative understanding of a QD-based CQED system.

\section{References}

1. D. Fattal et al., Phys. Rev. Lett. 92, 037904 (2004).

2. J. P. Reithmaier et al., Nature 432, 197-200 (2004).

3. C. Santori et al., Nature 419, 594-597 (2002).

4. K.H. Madsen et al., arXiv 1012.0740, to appear in Phys. Rev. Lett. (2011) 